\title{
Spectrophotometric and Photometric Behaviours of Be Stars: the Circumstellar Envelope Formation Mechanisms
}

\section{J. Zorec}

Institut d'Astrophysique de Paris, CNRS, 98 bis bd. Arago, F-75014 Paris, France

\author{
A.M. Hubert, A. Moujtahid \\ Observatoire de Paris-Meudon, DASGAL/UMR 8633 du CNRS, \\ F-92195 Meudon Principal Cedex, France
}

\begin{abstract}
.
A mechanism to create circumstellar envelopes in Be stars is proposed. The mechanism assumes a mass-loaded wind produced by ablated discrete stellar ejecta. The discrete mass ejections are suggested by the long-term spectrophotometric behaviours of Be stars and by their short and long-lived light outbursts discovered recently.
\end{abstract}

\section{Introduction}

The long-term spectrophotometric behaviours ( $\mathrm{SPh}$ ), as well as the short and long-lived light outbursts of Be stars are phenomena which are probably related to circumstellar envelope (CE) formation mechanisms. They may be the consequence of discrete mass ejections, which produce an irregular mass distribution in the circumstellar environment. The mass ejected may be sufficient to build up a CE. The interaction of the ejected layers with the stellar wind can lead to an enhanced mass-flow with the characteristics of a CE. Let us first briefly review some relevant observational facts.

\subsection{Long-term SPh behaviours}

Long-term SPh behaviours revealed by the observations, spread over more than 50 years of about 50 Be stars (Moujtahid et al. 1998), show that Be stars can be in three different, either permanent or transitory SPh phases. These phases are: 1) normal $\mathrm{SPh}$ phase (SPh-N), when the energy distribution looks like that of a B star without emission; 2) emission $\mathrm{SPh}$ phase (SPh-E), when the second component of the Balmer discontinuity (BD) due to the $\mathrm{CE}$ is in emission; 3 ) absorption SPh phase (SPh-A), when the second BD is in absorption. In a given $\mathrm{SPh}$ phase the slopes of correlations among the $\left(V, \Phi_{\mathrm{rb}}, D\right)$ parameters are single or double valued, depending on the object $\left(V=\right.$ visual apparent magnitude; $\Phi_{\mathrm{rb}}$ $=$ gradient of the Paschen continuum in $\mu \mathrm{m} ; D=$ total Balmer discontinuity in $d e x$ ). The characteristics of these correlations are different, depending on whether the stars are in a SPh-E or in a SPh-A phase. Any SPh phase can appear whatever the value of $V \sin i$, though the rather permanent $\mathrm{SPh}-\mathrm{A}$ phases 
are more frequent in Be stars with a high $V \sin i$. This suggests that regions of the $\mathrm{CE}$ near the central star responsible for the $\mathrm{SPh}$ several aspects should not be strongly flattened and that the $\mathrm{CE}$ covers somehow the polar regions of the star.

\subsection{Outbursts}

Ground-based photometric surveys (Mennickent et al. 1994, Sterken et al. 1996, Pavlovski et al. 1997, Percy et al. 1997 among others) allowed to detect light outbursts in Be stars. Recently Hubert \& Floquet (1998) and Hubert et al. (1999) using HIPPARCOS photometry have detected short (days, tens of days) and long-lived (year) outbursts in a very large number of Be stars. The light curves of "long-lived outbursts" show a gradual rise (100/300 days) followed by a slow decay.

\section{First interpretations}

Moujtahid et al. (1998) have shown that the various SPh phases correspond to different density and temperature structures of the CE near the central star and that the variation from one to another structure produces the $\mathrm{SPh}-\mathrm{E} \rightleftharpoons \mathrm{SPh}$ $\mathrm{N} \rightleftharpoons \mathrm{SPh}-\mathrm{A}$ transitions. They also show that the single valued $(\Delta V, \Delta D)$ relations in SPh-E and in SPh-A phases can be explained in terms of slight CE extent and optical depth changes. A stretching of the CE that decreases its optical depth favors the increase of the emission in a SPh-E phase. A shrinkage of a CE that is able to increase the CE optical depth above a given limit, produces the $\mathrm{SPh}$ characteristics of $\mathrm{SPh}-\mathrm{A}$ phases. In most cases the mean radii of $\mathrm{CE}$ regions producing SPh-E variations are $R_{\mathrm{e}} \lesssim 2-3 R_{*}$. Double valued $(\Delta V, \Delta D)$ relations in SPh-E phases can be explained by the ejection of layers having masses $M_{\mathrm{Sh}} \sim 10^{-10}-10^{-9} \times \mathrm{M}_{\odot}$ whose optical depth decreases with time (Moujtahid et al. 1999). If this decrease is due to an expansion of the layer, the amplitudes of $\Delta V$ and $\Delta D$ variations that compare with observations are reproduced by expansions which do not go further than few $R_{*}$. Some theoretical $(\Delta V, \Delta D)$ relations for different aspect angles $i$ are shown in Fig. 1. Fig. 1a: relations due to an expanding layer which has a constant ellipticity $E$; Fig 1 b: the expanding layer has a variable ellipticity ( $E=h / R_{\mathrm{e}} ; h=$ constant). Fig $1 \mathrm{c}$ shows single valued $S P h$ relations due to an ellipsoidal $C E$ with constant volume, where the optical depth increases with time. This optical depth behaviour can be due to a continuous stellar wind and/or frequent and less massive discrete ejections, as suggested by spectroscopic observations (Floquet et al. 1999). The electron scattering optical depth of the expanding layer measured in the polar direction, $\tau_{e}^{p}=1$ in Fig. 1a and Fig. 1b, indicates that the ejected mass is $M_{\mathrm{Sh}} \sim 10^{-10} \mathrm{M}_{\odot}$. Finally, the discrete mass ejections can also change a given physical structure of the CE enough to produce the single valued $\mathrm{SPh}$ variations as well as the $\mathrm{SPh}$ phase transitions.

In Hubert et al. (1999) the light outbursts were also interpreted as due to optical depth variation of ejected layers. The masses of these layers are the same as those implied in the long-term $\mathrm{SPh}$ variations. The ejected layers were assumed to expand in order for their optical depth to decrease enough to account for the observed light variations. 


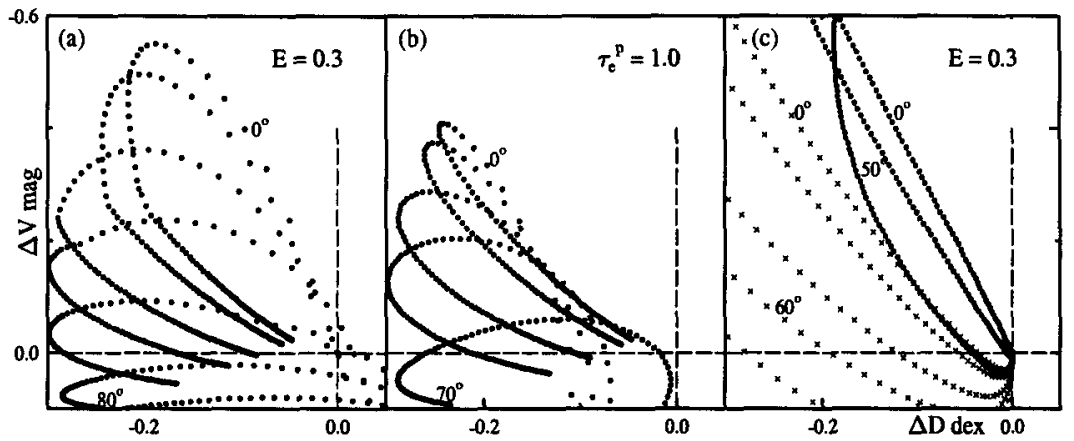

Figure 1. $\Delta V$ variations against $\Delta D$ for three $\mathrm{CE}$ configurations: a) expanding envelope with $\mathrm{E}=$ constant; $\mathrm{b}$ ) expanding $\mathrm{CE}$ with $\mathrm{E}$ variable; c) increasing density in a $\mathrm{CE}$ with constant volume.

The discrete mass ejection events which seem to provide consistent explanations for the SPh variations and for the light outbursts can, however, be followed and/or accompanied by a more or less variable but continuous massloss phenomenon. The interactions between the stellar wind and the ejected layers produce ablations of these ejecta, which favor a further decrease of their optical depth. On the other hand, the ablated gas enhances the mass-loaded flux around the central star in the nearest stellar environment, which can materialize a CE. In the next section we explore this possibility.

\section{Mass-loaded flows: a possible mechanism to create CE in Be stars}

The interaction of a high speed stellar wind with circumstellar gas produces a bubble with a flow pattern (Pickelner 1968, Dyson 1981) where an inward shock decelerates the stellar wind and an outwards directed shock accelerates the ambient gas.

The ejected layers, which were assumed to produce the light outbursts and the $\mathrm{SPh}$ variations, are ablated by the stellar wind so that they behave like mass sources in the circumstellar environment to produce a mass-loaded flux. The flow structure in the mass-loading core was described by Dyson \& Hartquist (1992) and Hartquist et al. (1994). The movement of its lower region is momentumdominated and the mass is mainly due to the ablated gas. There, the density distribution passes through a minimum at $R_{\min }$ and then it rises as $R^{4}$ until a critical radius $R_{\mathrm{c}}$. $R_{\mathrm{c}}$ can be assumed to be beyond the distribution of clumps, the residuals of ablated discrete ejecta. Outward this limit, the expansion is partially pressure dominated and the density decreases as $\mathrm{e}^{-\mathrm{M}^{2} / 2}$ (M is the Mach number).

Let us assume a typical Be star where the central star has a radius $R_{*}=$ $5 \mathrm{R}_{\odot}$, a mass-loss rate $M_{*}=10^{-10} \mathrm{M}_{\odot} \mathrm{yr}^{-1}$ and a wind velocity $V_{*}=500 \mathrm{~km}$ $\mathrm{s}^{-1}$. Following the interpretations of outbursts and $\mathrm{SPh}$ variations, we assume that the bulk of the CE mass is in a volume of a few stellar radii $\left(R_{\mathrm{e}} \sim 3 R_{*}\right)$. 
To produce Balmer emission lines we need $R_{\mathrm{e}} \sim 10 R_{*}$, but densities at such distances are quite low and do not change our estimate of the CE mass $M_{\mathrm{CE}}$. Depending on the geometry and the density distribution assumed in the CE and whether they are disc shaped or spherical, the total mass in the CE is roughly $10^{-10} \mathrm{M}_{\odot} \lesssim M_{\mathrm{CE}} \lesssim 10^{-8} \mathrm{M}_{\odot}$.

Using the important fact that the appearance of SPh-A and SPh-E phases is not aspect angle selective, to a good first order estimate we can adopt a spherical circumstellar mass environment near the central star. Hence, from relations derived by Dyson \& Hartquist (1992) and Hartquist et al. (1994), we estimate the mean mass injection rate from clumps $\dot{q}$ needed so that $R_{\min } \simeq R_{*}$. We thus obtain $\dot{q} \simeq 3 \dot{M}_{*} / 8 \pi R_{*}^{3} 2 \times 10^{-20} \mathrm{~g} \mathrm{~cm}^{-3} \mathrm{~s}^{-1}$. From a slightly more observational ground, the rate $\dot{q}$ can also be estimated using the expression for the radius of the expanding momentum-driven circumstellar bubble $R_{\mathrm{b}} \simeq R_{\mathrm{e}}$ at a time $t$. Adopting $t \sim \mathrm{yr}$ given by the characteristic time scale of the light decay in the observed long-lived outbursts of Be stars, we obtain $\dot{q} \simeq \dot{M}_{*} V_{*} t / R_{b}^{4}$ $\simeq 7 \times 10^{-20} \mathrm{~g} \mathrm{~cm}^{-3} \mathrm{~s}^{-1}$, which is consistent with the above estimate. Adopting $\bar{T}_{\mathrm{CE}} \simeq 10^{4} \mathrm{~K}$ as the mean temperature of the CE, we obtain $R_{\mathrm{c}}=\left(2 V_{*} / v_{t h}\right)^{1 / 3}$ $\simeq 4 R_{*}$. So, the mass gathered in the mass-loading core is $M_{\mathrm{LC}} \gtrsim 5 \pi \dot{q} R_{c}^{7} / 21 \dot{M}_{*} V_{*}$ $\simeq 10^{-10} \mathrm{M}_{\odot}$. This value compares with the lower limit of $M_{\mathrm{CE}}$ estimated above. As the masses of the ejected shells are $M_{\mathrm{Sh}} \sim 10^{-10}-10^{-9} \mathrm{M}_{\odot}$, it can be expected that a few discrete matter ejection events can rapidly increase $M_{\mathrm{LC}}$ up to the upper limit estimated for $M_{\mathrm{CE}}$.

We finally note that the temperature in the shock fronts can be high enough, $T \simeq(1 / 3)\left(V_{*} / v_{t h}\right)^{2} T_{\mathrm{CE}} \sim 5 \times 10^{6} \mathrm{~K}$, to produce ions like O VI, C IV, Si IV. The observed transitions of these superionized species in the far-UV could come from the backward directed front near the central star, where the column densities are expected to be higher.

\section{References}

Dyson, J.E. 1981, In: Investigating the Universe, (ed.) F.D. Kahn, Reidel Pub. Comp. p. 125

Dyson, J.E., Hartquist, T.W. 1992, Astro. Lett. and Comm. 28, 301

Floquet, M., Hubert, A.M., Hirata, R. et al. 1999, A\&A (to be submitted)

Hartquist, T.W., Dyson, J.E., Pettini, M. et al. 1986, MNRAS 221, 715

Hubert, A.M., Floquet, M. 1998, A\&A 335, 565

Hubert, A.M., Floquet, M., Zorec, J. 2000, "Short-lived and long-lived outburts in B and Be stars" (this issue)

Mennickent, R.E., Vogt, N., Sterken, C. 1994, A\&AS 108, 237

Moujtahid, A., Zorec, J., Hubert, A.M. 1998, A\&AS 129, 289

Moujtahid, A., Zorec, J., Hubert, A.M. 1999, A\&A 349, 151

Pavlowski, K., Harmanec, P., Božič, H. et al. 1997, A\&AS 125, 75

Percy, J.R., Harlow, J., Hayhoe, K.A.S. et al. 1997, PASP 109, 1215

Pickelner, S.B. 1986, Astrophys. Lett. 2, 97

Sterken, C., Vogt, N., Mennickent, R.E. 1996, A\&A 311, 579 